\title{
Modeling the consistency between the bony and facial chin outline in craniofacial superimposition
}

\author{
Carmen Campomanes-Alvarez ${ }^{1,2}$ Oscar Ibanez $^{1,2}$ Oscar Cordon $^{1,2}$ \\ ${ }^{1}$ Department of Computer Science and Artificial Intelligence, University of Granada, 18014 Granada, Spain \\ ${ }^{2}$ European Centre for Soft Computing, 33600 Mieres, Asturias, Spain
}

\begin{abstract}
Craniofacial superimposition is a forensic identification method involving the overlay of a skull over the available ante-mortem photographs of a candidate missing person face and the subsequent analysis of their anatomical correspondence. Within this process, the decision making stage focuses on determining the degree of support of being the same person or not based on the analysis of some criteria assessing the skull-face morphological correspondence. That decision is usually obtained in a non automatic and subjective way. We aim to automate the decision making process using computer vision and soft computing methods to assist the forensic anthropologist. In this work, we present a first approach to model one of the criteria followed by forensic experts: the analysis of the consistency of bony and facial chin outlines. We show some preliminary results over 82 skull-face overlay instances and discuss future research directions.
\end{abstract}

Keywords: Craniofacial superimposition, Decision making, Computer vision, Soft computing, Spatial relations

\section{Introduction}

Skeleton-based identification methods have been under continuous investigation within the forensic anthropology and odontology communities [1, 2]. Craniofacial superimposition (CFS) [3], one of the approaches in craniofacial identification $[4,5]$, is a representative technique of this kind. It involves the superimposition of a skull with a number of antemortem (AM) images of a missing person and the analysis of their morphological correspondence to determine if they belong to the same subject. Three consecutive stages have been distinguished for the whole CFS process in [6]: 1) Acquisition and processing of the skull (or skull 3D model) and the AM facial images, and the craniometric and facial landmark location; 2) skull-face overlay (SFO), which focuses on achieving the best possible superimposition of the skull and a single AM image of the missing person. This process is repeated for each available photograph, obtaining different overlays. Thus, SFO corresponds to what traditionally has been known as the adjustment of the skull size and its orientation with respect to the facial photograph $[3,7]$; and 3) decision-making. Based on the superimpositions achieved in the latter SFO stage, the degree of support of being the same person or not (exclusion) is determined by considering the different factors studying the relationship between the skull and the face: the morphological correlation, the matching between the corresponding landmarks according to the soft tissue depth, and the consistency between asymmetries.

There is a strong interest in designing automatic methods to support the forensic anthropologist to put CFS into effect [8]. In particular, the design of computer-aided CFS methods has experienced a boom over the past twenty years [9]. The most recent approaches consider the use of skull 3D models, as it is the case in the current contribution.

The works developed by authors such as [10, 11, $12,13,14]$ serve as examples of how computer algorithms can automate SFO and accommodate the uncertainty/fuzziness of some facial landmarks [15] and of the soft tissues [16, 17]. These methods represent a clear step forward since they have managed to reduce time and subjectivity inherent to manual approaches.

In the third stage, forensic experts evaluate spatial and morphological skull-face relationships focusing on certain regions that demonstrated to be more discriminative. The final decision is provided in terms of strong, moderate or limited support to the assertion that the skull and the facial image belong to the same person or not [18]. This is a subjective process that can depend on the expert's experience and the quantity and quality of the used materials. Hence, there is a need to help forensic anthropologists to take a more objective objective final decision. Our final aim is to design a decision support system based on the evaluation of the said spatial and morphological relations. This system will provide a numeric index as output, aiming to support to forensic anthropology to take the CFS final decision.

Computational methods in the fields of computer vision (CV) and soft computing ( $\mathrm{SC}$ ) can be extremely useful for the latter aim. Computer vision includes techniques for processing, analyzing, 
segmenting, and registering image data in an automatic way [19]. Meanwhile, SC is aimed for the design of intelligent systems to process uncertain, imprecise, and incomplete information [20]. Soft computing methods applied to real-world problems often offer more robust and tractable solutions than those obtained by more conventional mathematical techniques. Two of the main SC techniques are fuzzy logic (FL) and fuzzy set theory, which extend classical logic to provide a conceptual framework for knowledge representation under imprecision and the consequent uncertainty [21].

In this work, we take a first step to design a decision support system for CFS. To do so, we consider the modeling of one of the most discriminative criteria for assessing craniofacial correspondence, i. e. the morphological and spatial relationship between the bony and facial chin. Specifically, we have implemented a CV method aimed to measure how the chin facial shape follows the skull shape given the delineation of these regions in a particular SFO. That process involves the proper extraction of the two required chin curves (from the region given at hand) and the subsequent analysis of the relationship between them. In this first proposal we modeled the consistency of the bony and facial chin outlines using the spatial relation "along" presented in [22] and a shape similarity measure sensitive to rotation, the comparison between chain codes [23]. Each of them provides an index in the range $[0,1]$ that indicates how similar both shapes are.

This contribution is organized as follows: Section 2 reviews previous proposals dealing with forensic anthropology based on $\mathrm{CV}$ and SC, introduces our previous automatic SFO approach, and outlines the main issues related to the final decision making stage. Section 3 is devoted to explain our general methodological proposal for the development of a CFS decision support system while Section 4 focuses on the specific case study modeled in this work. Section 5 introduces the experimental setup, the corresponding results and their analysis. Finally, Section 6 outlines the conclusions and future works.

\section{Preliminaries}

\subsection{Computer Vision and Soft Computing Techniques in Forensic Anthropology}

Computational methods as $\mathrm{CV}$ and $\mathrm{SC}$ can be extremely useful for the automation of the decision making process. The guiding principle of these methods is perfectly adapted to the way in which reasoning and deduction have to be performed in forensic science. In fact, several successful applications of these techniques in forensic anthropology have been developed so far, such as age estimation [24, 25], skull 3D modeling [26], facial soft thickness prediction [27], facial identification [28], and skull 3D model simplification [29]. Within CFS, Evolu- tionary Algorithms (EAs) and fuzzy sets have being used to tackle SFO in an automatic way using EAs and fuzzy sets $[12,11,13,14]$. The following section 2.2 summarizes the latter SFO system since the SFO images analyzed in this work have been obtained using it.

\subsection{Automatic Skull-Face Overlay}

The SFO process requires positioning the skull in the same pose as the face in the photograph. From a $\mathrm{CV}$ point of view, the AM image is the result of the $2 \mathrm{D}$ projection of a real (3D) scene that was acquired by a particular (unknown) camera. In such a scene, the living was somewhere inside the camera field of view with a given pose [30].

The most natural way to face the SFO problem is to replicate that original scenario. To do so, a $3 \mathrm{D}$ model of the skull must be considered. The goal is to adjust its size and its orientation with respect to the head in the photograph [3]. In addition, the specific characteristics of the camera must also be replicated to reproduce the original as much as possible [30]. To do that, the skull 3D model is positioned in the camera coordinate system through geometric transformations, i.e. translation, rotation, and scaling which corresponds to the adjustment of the skull size and its orientation in the same angle as the face in the image $[3,31]$. Then, a perspective projection of the skull 3D model is performed onto the facial photograph.

In our automatic SFO procedure [11, 12, 13, 14], this approach is guided by a set of cranial and facial landmarks previously located by a forensic expert. An EA iteratively searches for the best geometric transformation, corresponding to 12 real-coded parameters in our model [11]. The method output corresponds to the solution providing the best matching between the two sets of landmarks.

Fuzzy landmarks in photographs are used to jointly deal with the imprecise landmark location and the coplanarity problem [12]. Besides, since the correspondence between facial and cranial landmarks is not always symmetrical and perpendicular [32], cranial landmarks are also modeled by fuzzy sets in [14], taking into account the available information concerning soft tissue depths $[16,17]$.

\subsection{Decision Making in Craniofacial Superimposition}

Once a SFO has been achieved, the main goal is to determine the degree of support that the skull and the face of the photograph belong to the same person or not. This degree of support is based on the consistency of the matching between the face and the skull but it is also influenced by the quality and quantity of the materials used (photographs and skull). A scale for a craniofacial matching evaluation has been recently defined by some of the most representative experts in craniofacial identification 
in [18]. Accordingly, the final decision is provided in terms of strong, moderate or limited support.

This decision is guided by different criteria studying the anatomical relationship between the skull and the face. According to the literature we can distinguish the following families of criteria for assessing the craniofacial correspondence:

1. Analysis of the consistency of the bony and facial outlines/morphological curves.

2. Assessment of the anatomical consistency by positional relationship.

3. Location and comparison lines to analyze anatomical consistency.

4. Evaluation of the consistency of the soft tissue thickness between corresponding cranial and facial landmarks.

Our long term goal is to automate the whole decision making process by modeling the former four sets of criteria using CV and SC techniques. The resulting system would give as a result a global degree of support of a CFS identification to assist the forensic anthropologist to take her/his decision. In this contribution, we take a first step in the design of such system by modeling a single criteria from the first family mentioned above.

Some automatic techniques have been proposed as decision support systems assisting the anthropologist in making the final identification decision. In $[33,34]$, k-th-order polynomial functions and Fourier harmonic analysis are applied to assess the fit between the outline of the skull and the face. In [35], the authors present a skull identification system, where the distance between the landmarks and the thickness of the soft tissue of the anthropometrical points are semi-automatically measured on the monitor for the assessment of the anatomical consistency between the digitized skull and face. The software also includes polynomial functions and Fourier harmonic analysis for evaluating the match of outlines. To extract the outline, gradient and threshold operations are used. A different approach is presented in [36], where an algorithm calculates the distance moved for crosses (that were manually marked by the expert in both face and skull radiograph images) and the respective mean in pixels.

These systems provide a generic value that do not take into account the spatial relation between skull and face. In addition, they do not integrate several criteria neither the quantity and quality of the materials to give the final decision.

\section{Methodology}

The decision making stage shows certain characteristics leading us to think on $\mathrm{CV}$ and fuzzy sets as powerful tools to automate it by developing:

- The proper extraction of curves or contours of a region in an automatic way.

- The modeling of the criteria for assessing the SFO relationship.
- The integration of different methods to evaluate a specific spatial skull-face relation.

- The aggregation of multiple degrees of support, each of them indicating the consistency between the skull and face guided by a particular criteria and from different facial regions.

- The integration of the quality and quantity of the materials within the decision process.

The need of fuzzy approaches is justified by the imprecision on the manual delineation of facial regions, but also to model the semantics of some anthropometrical relationships: "the prosthion lies posterior to the anterior edge of the upper lip", or "the nasion is higher than the nasal root", for example. In addition, they can provide a powerful tool for information fusion in order to take the final identification decision. In particular, fuzzy integral shows interesting capabilities to provide a support of the final decision by combining the individual sources, the expected worth of subsets of these sources, and the experts, knowledge on them. Thus, we aim to use fuzzy integral as a multifactorial way to analyze methods taking into account any variable (known or observed) that can be quantified to reach a decision (as done in previous approaches like [25]). Thanks to its use, the uncertainties and errors quantified along the whole process (e.g. the uncertainty in contours location) can be accumulated in order to consider their influence in the final decision.

As described in Section 2.3, one of the most important criteria used by forensic experts to assess whether the skull belong to a person is the consistency of the bony and facial outlines or morphological curves. Depending on the photograph view, these shapes have to satisfy a particular condition.

By analyzing the existing studies of this area, we have identified the three most common relations between the bony curves (B) and the face curves (F) used by forensic experts as follows:

- F follows B.

- $\mathrm{F}$ is consistent with $\mathrm{B}$.

- $\mathrm{F}$ is the mirror image of $\mathrm{B}$.

Regarding to their modeling the first two have similar effects. A visual example of them is shown in Fig 1. Notice that these kinds of relations are established in different facial regions, for example, the outline of the frontal bone follows the forehead outline and the chin outline is consistent with the mental outline.

To model the latter using CV, we first need to manually locate the regions under study in both the $3 \mathrm{D}$ skull and facial photograph. Then, the 3D regions are projected into the $2 \mathrm{D}$ image (using the resulting geometric transformation obtained in the SFO stage) to analyze their consistency with the corresponding facial contour. Of course, this task is affected by imprecision and subjectivity in the location of the different regions involved. 


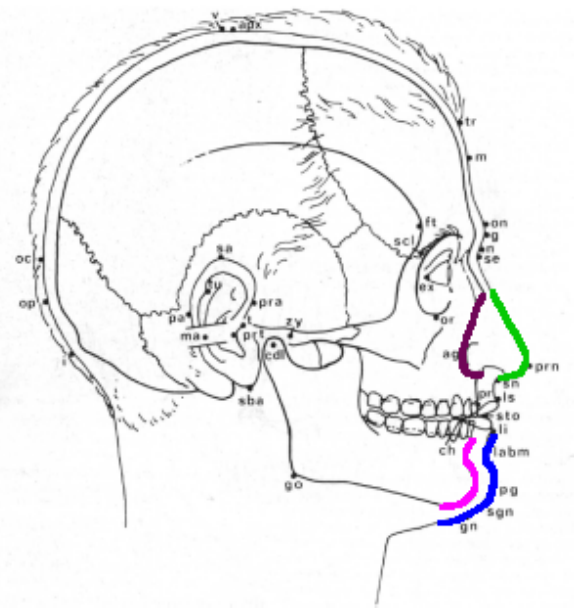

Figure 1: Example of two criteria used by forensics experts in lateral view to assess the consistency between bony and facial outlines. The chin facial curve follows the skull shape while the protruding part of the nose cartilage is the mirror image of the cartilage filling the pyriform aperture of the cranium.

In order to compare both curves there is a need to extract the corresponding contour of the skull region (the bony outline) under study (see Fig. 1). Automating contour extraction using $\mathrm{CV}$ techniques is not a trivial task and it could require a different approach depending on the region at hand, so it has to be studied for each specific case.

As a proof of concepts of the proposed methodological above we consider two different families of methods to model the " $F$ follows B" relation: the spatial relation along and shape similarity.

\subsection{Spatial Relation Along}

The spatial relation along give us the degree to which an object $A$ is along an object $B$. The considered approach is based on computing a degree of elongatedness of the region lying between $A$ and $B$, i.e. the between region [22]. Thus, it directly models the specific case of the "F follows B" relation by measuring whether the projected bone curve (a specific part of the projected bone region) follows its counterpart facial curve in the 2D facial image.

Two main steps have to be performed: Calculate the between region (noted by $\beta$ ) and measure how elongated is (thus defining the degree to which $A$ is along $B)$.

In [37] the authors present different methods to calculate the region between two objects. In this work we decide to use the model based on the convex hull of the union of the two objects. The between region can be calculated from the object contours using efficient algorithms, so the computational complexity is lower than other methods.

Once the between region is obtained, the alongness measure may be computed as

$$
\begin{gathered}
\alpha_{C_{1}}=f^{a}\left(P(\beta)^{2} / A(\beta)\right), \\
\alpha_{C_{2}}=f^{a}\left(P\left(C_{I N}\right)^{2} / A(\beta)\right),
\end{gathered}
$$

where $\mathrm{P}$ and $\mathrm{A}$ represent the perimeter and the area of $\beta$, and $C_{I N}$ is the inner contour that corresponds to those contours portions which belong to $\beta$. To normalize this measure between 0 and 1 , we use a sigmoid function as $f_{a}(x)=(1-$ $\exp (-a x)) /(1+\exp (-a x))$, taken from [22]. These measures $\alpha_{C_{1}}$ and $\alpha_{C_{2}}$ tend towards 1 as $\beta$ becomes more elongated. Absolute values can be changed by tuning parameter $a$ to enhance the differences depending on the application.

\subsection{Shape Similarity}

Similarity measures between shapes are usually employed to identify objects in images despite the different location, size, rotations or deformations [23]. There are many different similarity measures proposed in the specialized literature. They are obtained from a distance metric and thus are affected by the properties of such distance. Hence, there is a need to select the most appropriate one for the problem at hand to achieve a good performance.

A common way to study the similarity between two shapes consists of comparing both shapes represented by their features (using shape parameters or shape description techniques). Shape parameters are simple geometric features that can be used to describe shapes in a general way (i.e.: circularity, eccentricity, elliptic variance, convexity, etc.), but they can only discriminate shapes with large differences. Alternatively, there are many different image analysis methods, contour-based and regionbased, to measure the representation and description of a shape [38]. We will only focus on contourbased methods due to the impossibility to precisely locate the bony region within a facial photograph. In addition, these measures are usually employed to compare objects of the same kind but this is not our case. Firstly, there is an uncertain distance between both contours and, secondly, skull and face have different sizes since they are different objects. Besides, in the third stage, we work with the best SFO achieved in the previous step. Hence, large size differences between skull and face (scale variances) and high distances between skull and face curves (translation variances) are not common. As a consequence, translation and scale invariant measures are appropriate for our particular application. Besides, they must be sensitive to rotation and deformation changes.

In [39] the authors present a survey of the existing approaches for shape-based feature extraction, classified them according to their processing approaches:

- One-dimensional function for the shape representation.

- Polygonal approximation.

- Spatial interrelation feature.

- Moments.

- Scale-space methods. 
- Shape transforms domains.

Based on this overview, we have decided to use the chain code representation for our application, due to its low computational complexity as well as its interesting properties of being invariant to translation and sensitive to rotation changes.

Chain code is a common approach for representing contours. It describes an object by a sequence of unit-size line segments with a given orientation [38]. Freeman [40] introduced a chain code that describes the movement along a digital curve by using socalled 8-connectivity or 4-connectivity. The direction of each movement is encoded by the numbering scheme $\{i \mid i=0,1,2, \ldots, 7\}$ or $\{i \mid i=0,1,2,3\}$ denoting a counter-clockwise angle of $45^{\circ} \times i$ or $90^{\circ} \times i$ regarding the positive $x$-axis.

In our implementation, we compute the similarity between boundaries by comparing their chain codes as follows (using 8-connectivity):

$$
\alpha_{\text {chaincode }}=\sum_{i=1}^{N} d_{i} / N * 4
$$

where

$$
\begin{gathered}
d_{i}=\left\{\begin{array}{ll}
d_{i}^{*} & \text { if } d_{i}^{*} \leq 4 \\
8-d_{i}^{*} & \text { if } d_{i}^{*}>4
\end{array},\right. \\
d_{i}^{*}=|\operatorname{ch}(F)-\operatorname{ch}(B)|
\end{gathered}
$$

with $\operatorname{ch}(X)$ being the chain code representation of an object, $d_{i}$ the distance between two chain code representations, and $N$ the number of points of the objects. Notice that we normalize the final measure with respect to $N * 4$ since 4 is the largest distance between two chain codes using 8-connectivity (i.e., the opposite direction). This approach implies both curves must have the same number of points. To solve this problem a sampling method can be applied in one of them.

\section{Case Study: Chin Shapes}

Within the group of criteria that study the consistency of the bony and facial morphological curves, experts have stated that the chin outline has to be consistent with the mental outline. This relationship between bony and facial contours can be modeled with the spatial relation described in Section 3.

For the case at hand, we use a method that involves the convex hull of the union of the two objects in order to segment the contours. The first step consists of obtaining the region between both objects defined as [37]:

$$
\beta_{C H}(F, B)=C H(F \cup B) \cap F^{C} \cap B^{C}
$$

where $\mathrm{CH}(\mathrm{X})$ denotes the convex hull of $X$ and $X^{C}$ its complement. The basic idea of this method

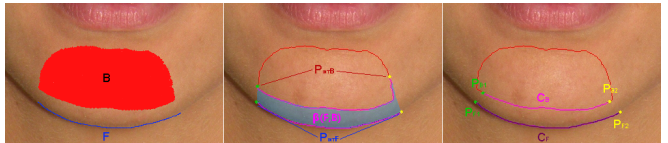

Figure 2: Chin contours segmentation. (a) Original regions, facial (F) and bony (B). (b) Convex hull $C H(F \cup B)$ with intersection points between $\beta(F, B)$ and $C H$. (c) Final contours with corresponding extreme points.

is that the contour of the between region is composed of an alternate sequence of line segments of the convex hull and the boundary of the two objects. The intersection points are the pairs of subsequent vertexes belonging to different objects. These points divide each contour into two portions (inner and outer contours). The inner contours correspond to the skull and face contours that we want to analyze (refer to Fig. 2 for an illustrative example). In some cases this method has a wrong behavior under some circumstances as depicted in Fig. 2 b. That problem is solved setting the intersection points of the bony contour as the nearest points to the corresponding intersection points of the facial contour.

Once the contour segmentation has been accomplished, we have to study whether the facial curve follows the bony curve. With this segmentation method, when both curves cross, it is not possible to extract the contours, so the result value will be zero. This result concurs with the fact that this is morphologically impossible. In this work, we propose to use the spatial relation along and a shape similarity method based on comparison between chain codes, as introduced in Section 3.

\section{Experiments}

Some experiments have been developed to analyze the behavior of our two automatic methods to measure the morphological correspondence between bony and facial chin shapes. Both positive and negative identification cases have been considered.

\subsection{Experimental Setup}

The experimental design involves 82 problem instances. The dataset is composed by 32 SFOs corresponding to four female skulls (FS1-4) compared with eight photographs, and 50 SFOs corresponding to five male skulls (MS1-5) compared with 10 photographs. The used skull models correspond to nine cases of living (from Spain and Italy) for each of which two different photographs are available in lateral and frontal poses. Thus, each female skull has a positive case and three negative cases and each male skull has a positive case and four negative cases. The SFOs have been obtained by our automatic method in [14] using parameter values reported in that contribution.

The morphological correspondence between chin shapes is evaluated using the two proposed methods for each photograph. Then, the obtained values 

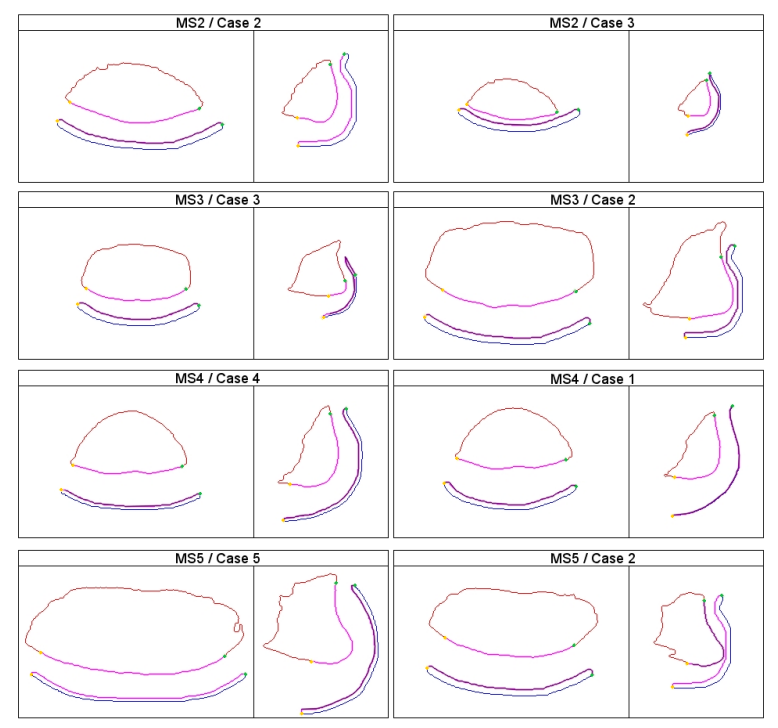

Figure 3: Visual results of some cases. MS2 and MS5 are wrongly classified by spatial relation "along" but positive cases (case two and five respectively) have a better consistency between bony and facial shapes than the wrongly classified instances (case three and two respectively). Comparison between chain codes ranks case two and one in the first position for the third and forth skull but this is visually concordant.

\begin{tabular}{|c|c|c|c|c|}
\hline & Case 1 & Case 2 & Case 3 & Case 4 \\
\hline \multirow{2}{*}{ FS1 } & $\mathbf{0 . 8 3 ( 1 )}$ & $0.45(4)$ & $0.49(3)$ & $0.50(2)$ \\
& $\mathbf{0 . 8 7}(\mathbf{1})$ & $0.42(4)$ & $0.42(3)$ & $0.43(2)$ \\
\hline \multirow{2}{*}{ FS2 } & $0.00(2)$ & $\mathbf{0 . 6 8 ( 1 )}$ & $0.00(2)$ & $0.00(2)$ \\
& $0.00(2)$ & $\mathbf{0 . 8 6 ( 1 )}$ & $0.00(2)$ & $0.00(2)$ \\
\hline \multirow{2}{*}{ FS3 } & $0.50(2)$ & $0.43(3)$ & $\mathbf{0 . 7 9 ( 1 )}$ & $0.00(4)$ \\
& $0.43(3)$ & $0.44(2)$ & $\mathbf{0 . 8 7}(\mathbf{1})$ & $0.00(4)$ \\
\hline \multirow{2}{*}{ FS4 } & $0.48(2)$ & $0.37(4)$ & $0.48(3)$ & $\mathbf{0 . 7 6 ( 1 )}$ \\
& $0.43(2)$ & $0.42(4)$ & $0.43(3)$ & $\mathbf{0 . 8 5}(\mathbf{1})$ \\
\hline
\end{tabular}

Table 1: Results for the measurement of the morphological correspondence between chin contours obtained using the spatial relation "along" (above) and comparison between chain codes (below) for female cases. The corresponding rankings are shown in brackets.

for the frontal and lateral view images of the same person are aggregated using an arithmetic mean. Thus, each case will have a degree of support that indicates how the shape of the facial chin is consistent with the bony chin. Like this, we can establish a ranking of the possible candidates for each skull based only on the chin consistency.

\subsection{Results Obtained}

Table 1 shows the resulting values for the measurement of the morphological correspondence between chin shapes using the two proposed methods in female cases. Analogous results for male cases are shown in Table 2.

In view of the results in Table 1 , both methods can discriminate correctly the female cases. In every case, the actual candidate is classified as the right decision, i.e., it is ranked first in the ordering. In the positive cases (values of the table diagonal) comparison between chain codes provides higher values than those of the spatial relation "along".

Nevertheless, in male cases, none of the proposed

\begin{tabular}{|l|c|c|c|c|c|}
\hline & Case 1 & Case 2 & Case 3 & Case 4 & Case 5 \\
\hline \multirow{2}{*}{ MS1 } & $\mathbf{0 . 8 3 ( 1 )}$ & $0.41(4)$ & $0.49(2)$ & $0.43(3)$ & $0.00(5)$ \\
& $\mathbf{0 . 8 6}(\mathbf{1})$ & $0.43(2)$ & $0.41(4)$ & $0.43(3)$ & $0.00(5)$ \\
\hline \multirow{2}{*}{ MS2 } & $0.44(4)$ & $\mathbf{0 . 8 1 ( 2 )}$ & $0.93(1)$ & $0.00(5)$ & $0.50(3)$ \\
& $0.42(4)$ & $\mathbf{0 . 8 9}(\mathbf{1})$ & $0.87(2)$ & $0.43(3)$ & $0.37(5)$ \\
\hline \multirow{2}{*}{ MS3 } & $0.79(3)$ & $0.87(2)$ & $\mathbf{0 . 8 9}(\mathbf{1})$ & $0.48(5)$ & $0.49(4)$ \\
& $0.83(2)$ & $0.88(1)$ & $\mathbf{0 . 7 0}(\mathbf{3})$ & $0.37(5)$ & $0.43(4)$ \\
\hline \multirow{2}{*}{ MS4 } & $0.66(2)$ & $0.34(5)$ & $0.45(4)$ & $\mathbf{0 . 6 9}(\mathbf{1})$ & $0.50(3)$ \\
& $0.84(1)$ & $0.42(5)$ & $0.43(4)$ & $\mathbf{0 . 8 3}(\mathbf{2})$ & $0.44(3)$ \\
\hline \multirow{2}{*}{ MS5 } & $0.39(5)$ & $0.86(1)$ & $0.40(3)$ & $0.40(4)$ & $\mathbf{0 . 8 2}(\mathbf{2})$ \\
& $0.42(5)$ & $0.83(2)$ & $0.46(4)$ & $0.43(3)$ & $\mathbf{0 . 9 0}(\mathbf{1})$ \\
\hline
\end{tabular}

Table 2: Results for the measurement of consistency between chin contours obtained using the spatial relation "along" (above) and comparison between chain codes (below) for male cases. The corresponding rankings are shown in brackets.

methods manage to rank the positive candidates in the correct order (Table 2). The spatial relation "along" fails in the classification of the second and fifth instances, setting the right candidate in the second position. The comparison between chain codes wrongly classifies instances three and four, setting them in third and second position, respectively. To develop a deeper analysis of that behavior, the corresponding visual results are shown in Fig. 3. In the second skull (MS2) the method based on the spatial "along" provides a higher degree for the third case than for the second one (the correct one). This result does not concur with the visual analysis, since the second case shows a better consistency between bony and facial shapes, as can be seen in the first row of Fig. 3. The same happens for the fifth skull (MS5), where the spatial relation "along" gives a higher value for the second case than for the positive case (case five), as depicted in the last row of Fig. 3. However, the cases where the comparison between chain codes fail (MS3 and MS4), have a good consistency between bony and facial shapes, similar in the right instances and in the wrong ones (second and third rows of Fig. 3). Hence, the method performs appropriately in those cases.

\section{Conclusions and Future Works}

In this work, we have introduced a new methodology to model spatial and morphological relations between skull and face. We have proposed two different methods to measure the consistency between bony and facial chin shapes, which have been validated in 82 SFOs. The comparison between chain codes has showed a better performance than the spatial relation "along", which provided low degrees for positive cases and failed in the classification of some visually good instances. However, we have shown that using only one region (i.e. the chin) to analyze the consistency between the skull and the face is not enough, because some negative cases can also show a good consistency in this isolated part than the positive cases (and probably not in other regions).

Therefore, there is a need to analyze and aggregate more morphological criteria within the decision making stage. This way, future research directions include the modelization of other criteria for 
craniofacial anatomical assessment. Meanwhile, we plan to study other techniques to model the consistency between bony and facial shapes using similarity measures. Then, a degree of confidence for each of them can be obtained analyzing their performance over a ground truth dataset [41]. Multifactorial methods have been shown to produce better results than single indicators methods [25], so, the integration of these techniques can provide a most confident value to measure spatial relations between bony and facial shapes. Finally, other assessment methodologies including the quality and quantity of the used materials will also be studied for this goal.

\section{Acknowledgements}

This work has been supported by the Spanish Ministerio de Economía y Competitividad under the SOCOVIFI2 project (refs. TIN2012-38525-C02-01/02, http://www.softcomputing.es/socovifi/), the Andalusian Dept. of Innovación, Ciencia y Empresa under project TIC2011-7745, including European Development Regional Funds (EDRF), the GENIL programme of the CEI BioTic Granada (project PYR-2014.14), and the Principality of Asturias Government under the project with reference CT1355 and "Programa Asturias 2014-2015" under the project CT14-05-2-05. Mrs. C. CampomanesÁlvarez's work has been supported by Spanish MECD FPU grant AP-2012-4285. Dr. Ibáñez's work has been supported by Spanish MINECO Juan de la Cierva Fellowship JCI-2012-15359. Authors are also grateful to Caroline Wilkinson for supporting them with her deep craniofacial identification knowledge.

\section{References}

[1] K. R. Burns and J. Wallington. Forensic anthropology training manual. Prentice Hall Saddle River, NJ, 1999.

[2] C. Cattaneo. Forensic anthropology: developments of a classical discipline in the new millennium. Forensic Sci Int, 165(2):185-193, 2007.

[3] M. Yoshino. Craniofacial superimposition. In C Wilkinson and C Rynn, editors, Craniofacial Identification, pages 238-253. University Press, Cambridge, 2012.

[4] W. A. Aulsebrook, M. Y. Iscan, J. M. Slabbert, and P. Beckert. Superimposition and reconstruction in forensic facial identification: a survey. Forensic Sci Int, 75:101-120, 1995.

[5] C. N. Stephan. Craniofacial identification: Techniques of facial approximation and craniofacial superimposition. In S Blau and DH Ubelaker, editors, Handbook of Forensic Anthropology and Archaeology, volume 25, pages 304321. Left Coast Press, Walnut Creek, 2009.
[6] S. Damas, O. Cordón, O. Ibáñez, J. Santamaría, I. Alemán, M. Botella, and F. Navarro. Forensic identification by computer-aided craniofacial superimposition: a survey. ACM Computing Surveys, 43(4):27, 2011.

[7] S. Al-Amad, M. McCullough, J. Graham, J. Clement, and A. Hill. Craniofacial identification by computer-mediated superimposition. J Forensic Odontostomatol, 24:47-52, 2006.

[8] D. H. Ubelaker. A history of smithsonian-FBI collaboration in forensic anthropology, especially in regard to facial imagery. Forensic Sci Communications, 2(4), 2000.

[9] M. I. Huete, T. Kahana, O. Ibáñez, and C. Wilkinson. Past, present, and future of cfs: literature and international surveys. Legal Medicine, 2015. In press.

[10] B. A. Nickerson, P. A. Fitzhorn, S. K. Koch, and M. Charney. A methodology for nearoptimal computational superimposition of twodimensional digital facial photographs and three-dimensional cranial surface meshes. $J$ Forensic Sci, 36(2):480-500, 1991.

[11] O. Ibáñez, O. Cordón, S. Damas, and J. Santamaría. An experimental study on the applicability of evolutionary algorithms to craniofacial superimposition in forensic identification. Inf Sci, 79:3998-4028, 2009.

[12] O. Ibáñez, O. Cordón, S. Damas, and J. Santamaría. Modeling the skull-face overlay uncertainty using fuzzy sets. IEEE Trans Fuzzy Syst, 16:946-959, 2011.

[13] O. Ibáñez, O. Cordón, and S. Damas. A cooperative coevolutionary approach dealing with the skull-face overlay uncertainty in forensic identification by craniofacial superimposition. Soft Comput, 18:797-808, 2012.

[14] B. R. Campomanes-Álvarez, O. Ibáñez, C. Campomanes-Álvarez, S. Damas, and O. Cordón. Modeling the facial soft tissue thickness for automatic skull-face overlay. Technical Report AFE-2014-05, European Centre for Soft Computing.

[15] B. R. Campomanes-Álvarez, O. Ibáñez, F. Navarro, I. Alemán, O. Cordón, and S. Damas. Dispersion assessment in the location of facial landmarks on photographs. Int $J$ Legal Med, 129(1):227-236, 2015.

[16] C. N. Stephan and E. K. Simpson. Facial soft tissue depths in craniofacial identification (part i): an analytical review of the published adult data. J Forensic Sci, 53:1257-1272, 2008.

[17] C. N. Stephan and E. K. Simpson. Facial soft tissue depths in craniofacial identification (part ii): an analytical review of the published subadult data. J Forensic Sci, 53:1273-1279, 2008.

[18] S. Damas, C. Wilkinson, T. Kahana, E. Veselovskaya, A. Abramov, R. Jankauskas, P.T. Jayaprakash, E. Ruiz, F. Navarro, M.I. Huete, E. Cunha, F. Cavalli, J. Clement, 
P. Leston, F. Molinero, T. Briers, F. Viegas, K. Imaizumi, D. Humpire, and O. Ibáñez. Study on the performance of different craniofacial superimposition approaches (ii): best practices proposal. Forensic Sci Int, 2015. In press.

[19] M. Sonka, V. Hlavac, and R. Boyle. Image processing, analysis, and machine vision. Cengage Learning, 2014.

[20] P. P. Bonissone. Soft computing: the convergence of emerging reasoning technologies. Soft Comput, 1:6-18, 1997.

[21] L. A. Zadeh. Soft computing and fuzzy logic. IEEE Softw, 11:48-56, 1994.

[22] C. M. Takemura, R. Cesar Jr, and I. Bloch. Fuzzy modeling and evaluation of the spatial relation "Along". In Progress in Pattern Recognition, Image Analysis and Applications, pages 837-848. Springer, 2005.

[23] R. C. Veltkamp. Shape matching: Similarity measures and algorithms. In Shape Modeling and Applications, SMI 2001 International Conference on., pages 188-197. IEEE, 2001.

[24] S. Aja-Fernández, R. de Luis-Garcı, M. A. Martın-Fernández, and C. Alberola-López. A computational tw3 classifier for skeletal maturity assessment. a computing with words approach. J Biomed Inform, 37(2):99-107, 2004.

[25] M. F. Anderson, D. T. Anderson, and D. J. Wescott. Estimation of adult skeletal age-atdeath using the sugeno fuzzy integral. Am J Phys Anthropol, 142(1):30-41, 2010.

[26] J. Santamaría, O. Cordón, S. Damas, J. M. García-Torres, and A. Quirin. Performance evaluation of memetic approaches in $3 d$ reconstruction of forensic objects. Soft Comput, 13:883-904, 2009.

[27] Q. H. Dinh, T. C. Ma, T. T. Nguyen, D. T. Nguyen, et al. Facial soft tissue thicknesses prediction using anthropometric distances. In New Challenges for Intelligent Information and Database Systems, pages 117-126. Springer, 2011.

[28] D. De Angelis, R. Sala, A. Cantatore, M. Grandi, and C. Cattaneo. A new computerassisted technique to aid personal identification. Int J Legal Med, 123(4):351-356, 2009.

[29] B. R. Campomanes-Álvarez, O. Cordón, and S. Damas. Evolutionary multi-objective optimization for mesh simplification of $3 d$ open models. Integr Comput-Aid E, 20(4):375-390, 2013.

[30] B. R. Campomanes-Álvarez, O. Ibáñez, F. Navarro, M. Botella, S. Damas, and O. Cordón. Computer vision and soft computing for automatic skull-face overlay in craniofacial superimposition. Forensic Sci Int, 245:7786, 2014.

[31] T. W. Fenton, A. N. Heard, and N. J. Sauer. Skull-photo superimposition and border deaths: Identification through exclusion and the failure to exclude. J Forensic Sci, 53(1):3440, 2008.

[32] G. M. Gordon and M. Steyn. An investigation into the accuracy and reliability of skullphoto superimposition in a south african sample. Forensic Sci Int, 216:198.e1-6, 2012.

[33] P. V. Delfino, M. Colonna, E. Vacca, F. Potente, and F. Jr. Introna. Computer-aided skull/face superimposition. The American journal of forensic medicine and pathology, 7(3):201-hyhen, 1986.

[34] D. V. Pesce, E. Vacca, F. Potente, T. Lettini, and M. Colonna. Shape analytical morphometry in computer-aided skull identification via video superimposition. Iscan $M Y$, Helmer RP. Forensic analysis of the skull: craniofacial analysis, reconstruction, and identification. New York: Wiley-Liss, 1993.

[35] M. Yoshino, H. Matsuda, S. Kubota, K. Imaizumi, S. Miyasaka, and S. Seta. Computer-assisted skull identification system using video superimposition. Forensic science international, 90(3):231-244, 1997.

[36] A. Ricci, G. L. Marella, and M. A. Apostol. A new experimental approach to computer-aided face/skull identification in forensic anthropology. The American journal of forensic medicine and pathology, 27(1):46-49, 2006.

[37] I. Bloch, O. Colliot, and R. M. Cesar. On the ternary spatial relation" between". Systems, Man, and Cybernetics, Part B: Cybernetics, IEEE Transactions on, 36(2):312-327, 2006.

[38] D. Zhang and G. Lu. Review of shape representation and description techniques. Pattern Recogn, 37(1):1-19, 2004.

[39] M. Yang, K. Kpalma, J. Ronsin, et al. A survey of shape feature extraction techniques. Pattern Recogn, pages 43-90, 2008.

[40] H. Freeman. On the encoding of arbitrary geometric configurations. Electronic Computers, IRE Transactions on, (2):260-268, 1961.

[41] O. Ibáñez, F. Cavalli, B. R. CampomanesÁlvarez, C. Campomanes-Álvarez, A. Valsecchi, and M. I. Huete. Ground truth data generation for skull-face overlay. Int $J$ of Legal Med, pages 1-13, 2014. 